\title{
BIBLIOGRAPHY
}

1. M. L. Curtis, Cartesian products with intervals, Proc. Amer. Math. Soc. 12 (1961), 819-820.

2. L. C. Glaser, Contractible complexes in $S^{n}$, Proc. Amer. Math. Soc. 16 (1965), (to appear).

3. B. Mazur, A note on some contractible 4-manifolds, Ann. of Math. 73 (1961), 221-228.

4. V. Poenaru, Les decompositions de l'hypercube en produit topologique, Bull. Soc. Math. France 88 (1960), 113-119.

5. T. Price, Equivalence of embeddings of $k$-complexes in Euclidean $n$-space for $n \leqq 2 k+1$, Abstract 614-121, Notices Amer. Math. Soc. 11 (1964), 564.

6. J. H. C. Whitehead, Simplicial spaces, nuclei and m-groups, Proc. London Math. Soc. 45 (1939), 243-327.

7. E. C. Zeeman, On the dunce hat, Topology 2 (1964), 341-358.

RICE UNIVERSITY

\section{EXTREMA CONCERNING POLYGONS IN SPACE-TIME}

BY LOUIS V. QUINTAS AND FRED SUPNICK

Communicated by Victor L. Klee, August 6, 1965

1. Consider an astronomer and his observation field, i.e., the set of observable (light or radio) signal emitting loci of the universe. Let the observation field be ordered by attaching a date to each observable locus indicating the time in the history of the universe that the signal was emitted from its source. Whereas both the astronomer and his observation field age with time, the observations of the astronomer may trace a sequence of loci whose time labels proceed forward or backward in time.

Consider now, a finite set $S$ of events in $L^{n}, n$-dimensional spacetime $^{1}(n \geqq 2)$. A rectilinear world line segment with endpoints in $S$ will be called a rectilinear connection in $S$ and a set of rectilinear connections which form a polygon with vertex set $S$ a polygonal connection of $S$. The clock time of a polygonal connection is defined to be the sum of all the time separations ${ }^{2}$ of its rectilinear connections. A polygonal connection having either the least or the greatest clock time of all possible "circuit states," i.e., all possible polygonal connections of

\footnotetext{
${ }^{1}$ Riemannian $n$-space having fundamental form $\Phi=\left(d x^{1}\right)^{2}+\cdots+\left(d x^{n-1}\right)^{2}$ $-(d t)^{2}$.

2 The time separation of a rectilinear connection with endpoints $E_{u}:\left(x_{u}^{1}, \cdots, x_{u}^{n-1}, t_{u}\right)$ and $E_{v}:\left(x_{v}^{1}, \cdots, x_{v}^{n-1}, t_{v}\right)$ is equal to $\left[\left(t_{v}-t_{v}\right)^{2}-\sum_{i=1}^{n-1}\left(x_{v}^{i}-x_{u}^{i}\right)^{2}\right]^{1 / 2}$ and will be denoted by $s\left(E_{w} E_{v}\right)$.
} 
$S$, will be called extreme with respect to $S$. In [1] criteria are established, which, if satisfied by a set $S$ of events in $L^{n}$, enables one to immediately obtain extreme polygonal connections of $S$. These results are summarized in the following section.

2. A set $S$ of events is called a timelike distribution, if each event of $S$ is in the interior of the time cone of every other event in $S$.

A timelike distribution $S$ in $L^{n}(n \geqq 2)$ is said to satisfy the Four Point Condition, if the events of $S$ can be labeled $P_{1}, P_{2}, \cdots, P_{k}$ so as to satisfy the following condition:

For all sets of integers $\{a, b, c, d\}$ such that $1 \leqq a<b<c<d \leqq k$ it follows that

$$
s\left(P_{a} P_{b}\right)+s\left(P_{c} P_{d}\right) \leqq s\left(P_{a} P_{c}\right)+s\left(P_{b} P_{d}\right) \leqq s\left(P_{a} P_{d}\right)+s\left(P_{b} P_{c}\right) .
$$

Basic Lemma. If a timelike distribution $S$ of $k$ events in $L^{n}(n \geqq 2)$ satisfies the Four Point Condition, then the polygonal connections

$$
\left[\cdots P_{7} P_{5} P_{3} P_{1} P_{2} P_{4} P_{6} \cdots\right]
$$

and

$$
\left[\cdots P_{k-5} P_{5} P_{\kappa-3} P_{3} P_{k-1} P_{1} P_{k} P_{2} P_{k-2} P_{4} P_{k-4} P_{6} \cdots\right]
$$

have respectively the least and the greatest clock time of the set of all possible polygonal connections of $S$.

The proof of this lemma is identical to that of Theorem III [2, p. 181]. The latter was proven in a Euclidean setting, but it is directly verified that this is not necessary.

In $1964 \mathrm{M}$. Lerman obtained the result (unpublished) that, if a set of $k$ noncollinear points in the Euclidean plane satisfies the Four Point Condition, i.e., the hypothesis of Theorem III [2], then $k \leqq 8$. This is contrasted with the fact that in $L^{2}$, for any $k \geqq 4$, it is possible to select $k$ noncollinear events and label them so as to satisfy (2.1). E.g., any $k$ events which lie on a convex timelike arc (in $L^{2}$ ) can so be labeled (cf. Theorem 4 below). Higher dimensional curves having this property are constructed in [1]. A still wider class of event distributions in $L^{n}(n \geqq 2)$ which satisfy the Four Point Condition is given in Theorem 2 below.

Our first theorem consists of a necessary and sufficient condition that a timelike distribution satisfy the Four Point Condition.

Theorem 1. Let $S=\left\{E_{1}, E_{2}, \cdots, E_{k}\right\} \quad\left(t_{1}<t_{2}<\cdots<t_{k} ; k \geqq 4\right)$ denote a timelike distribution in $L^{n}(n \geqq 2)$. Then, $S$ satisfies the Four Point Condition if and only if at least one of the following four labelings of $S\left\{P_{1}, P_{2}\right\}=\left\{E_{1}, E_{2}\right\}, P_{i}=E_{i}(i=3,4, \cdots, k-2)$, and $\left\{P_{k-1}, P_{k}\right\}$ $=\left\{E_{k-1}, E_{k}\right\}$ is a labeling for which (2.1) is satisfied. 
REMARK. Since there are only four labelings to check, Theorem 1 yields an effective computational method for determining whether or not a timelike distribution in $L^{n}(n \geqq 2)$ satisfies the Four Point Condition.

Let $U, V$, and $W$ denote three events in $L^{n}(n \geqq 2), F(W)$ the interior of the future time cone of $W$, and

$$
R(U, V ; W)=\{E \in F(W): s(U W)-s(V W) \leqq s(U E)-s(V E)\} .
$$

TheOREM 2. Let $S=\left\{E_{1}, E_{2}, \cdots, E_{k}\right\}(k \geqq 4)$ denote a set of events in $L^{n}(n \geqq 2)$ such that for $t=2,3, E_{t} \in F\left(E_{t-1}\right)$ and for $t=4,5, \cdots, k$, $E_{t} \in \cap R\left(E_{i}, E_{j} ; E_{t-1}\right)(1 \leqq i<j<t-1)$, then $S$ is a timelike distribution which satisfies the Four Point Condition.

In the next theorem we define a class of event distributions which includes the class of event distributions given in Theorem 2. For each $S$ in this class we are able to determine a minimal polygonal connection.

Theorem 3. Let $S=\left\{E_{1}, E_{2}, \cdots, E_{k}\right\}(k \geqq 4)$ denote a set of events in $L^{n}(n \geqq 2)$ such that for $t=2,3, E_{t} \in F\left(E_{t-1}\right)$ and for $t=4,5, \cdots, k$,

$$
E_{t} \in \cap R\left(E_{i-3}, E_{i-2} ; E_{i-1}\right) \quad(i=4,5, \cdots, t),
$$

then

$$
\left[\cdots E_{7} E_{5} E_{3} E_{1} E_{2} E_{4} E_{8} \cdots\right]
$$

has the least clock time of the set of all polygonal connections of $S$.

The following lemma and Theorem 4 below state some geometric properties of event distributions in $L^{2}$ which satisfy the Four Point Condition.

Lemma. If the events of a noncollinear timelike distribution of events in $L^{2}$ fall on the boundary $B$ of their convex hull and are labeled so as to satisfy (2.1), then the labeling must be cyclic with respect to $B$.

THEOREM 4 . Let $k$ events $(k \geqq 4)$ of a timelike distribution of events in $L^{2}$ fall on the boundary $B$ of their convex hull. Let $P, Q, R$, and $S$ denote the events with the minimal, next to minimal, next to maximal, and maximal $t$-coordinates respectively. Then, the $k$ events satisfy the Four Point Condition if and only if $B-\{P, S\}$ consists of one component or one of its two components contains at most two events from among the $k$ given events and if an event is in this component, then that event is either $Q$ or $R$.

A feature of $L^{n}$ is the existence of rectilinear connections having time separation equal to zero (world lines of photons). This gives rise to the consideration of a special class of minimal polygonal connec- 
tions, namely those which have clock time equal to zero. We call these, zero polygonal connections. An event distribution $S$ is said to be zero separated, if each pair of events in $S$ constitutes the endpoints of a polygonal path consisting of rectilinear connections of $S$ each of which have time separation equal to zero. We note that the vertex set of a zero polygonal connection is zero separated, but not conversely.

Let $C(E)$ denote the light cone centered at $E$, i.e., the boundary of the time cone of the event $E$.

TheOREM 5. Let $S=\left\{E_{1}, E_{2}, \cdots, E_{k}\right\}(k \geqq 4)$ denote a set of events in $L^{n}(n \geqq 2)$ which is zero separated and such that for each $i=1,2, \ldots$, $k$ the set $C\left(E_{i}\right) \cap S$ contains exactly three events, then there exists a unique zero polygonal connection of $S$.

\section{REFERENCES}

1. L. V. Quintas and F. Supnick, Extrema in space-time, Canad. J. Math. (to appear).

2. F. Supnick, Extreme Hamiltonian lines, Ann. of Math. (2) 66 (1957), 179-201.

ST. JOHN's UNIVERSITY, New York AND The City University of New York 